\title{
Stereoscopic Imaging of Transverse Detonations in Diffraction
}

\section{F. Pintgen ${ }^{1 *}$ and J.E. Shepherd ${ }^{2}$}

${ }^{1}$ Energy and Propulsion Technologies Laboratory

General Electric Global Research

Niskayuna 12309, NY

USA

${ }^{2}$ Graduate Aeronautical Laboratories

California Institute of Technology

Pasadena 91125, CA

USA

* Corresponding Author, pintgen@ge.com

Manuscript submitted for consideration of publication in:

Journal of Flow Visualization and Image Processing

Date: February 2, 2007 


\section{Abstract}

Diffraction of gaseous detonations has received considerable attention for many years, yet there is limited understanding of the failure and initiation phenomena due to the complex coupling between the combustion and the fluid dynamics. A variety of optical techniques such as streak imaging, open shutter photography, high-speed schlieren imaging, and, more recently, planar laser induced fluorescence (PLIF) has been used to visualize the diffraction process in detonations. To overcome the integrating nature of these visualization techniques and also allow for sooted foil records, many diffraction experiments in the past were carried out in narrow channels, studying detonation transition from planar to cylindrical geometry. The experimental investigation on spherically diffracting detonations described in this paper uses stereoscopic image reconstruction of the transverse detonations. The aim is to obtain further insight into the transverse detonations, which are the re-coupling phenomena identified to occur in the critical diffraction regime following a re-initiation event. The $3-\mathrm{D}$ reconstruction technique visualizes the transverse detonation as defined by the volume in space with high luminosity. The reconstruction technique is based on gradients, in contrast to those techniques based on target points as used, for example, in 3-D particle image velocimetry. Together with a simultaneously obtained schlieren image, the location of the transverse detonation could be determined to be just below the shock surface.

\section{Introduction}

If a detonation wave, propagating in a rigid tube or channel, suddenly emerges into an unconfined volume, the planar wave will diffract and transform into an approximately spherical or, in the case of a narrow channel, cylindrical wave (Fig 1a). The detonation diffraction process has been widely studied $[1,3,5,7,9,10]$ in the context of both applications and as a scientific experiment. Depending on the mixture parameters and the geometrical dimensions, transition from planar to spherical geometry may result in detonation failure or initiation. In the case of detonation failure, the leading shock wave decouples from the reaction front, Fig. 1b; this is the sub-critical regime. If the detonation transitions successfully, the shock and reaction zone remain coupled and re-initiation phenomena can occur (Fig 1c). A detailed understanding of the diffraction process is of importance for the combustion community as it gives insight into the essential mechanisms of initiation and failure, such as unsteadiness, wave curvature, and the role of the 


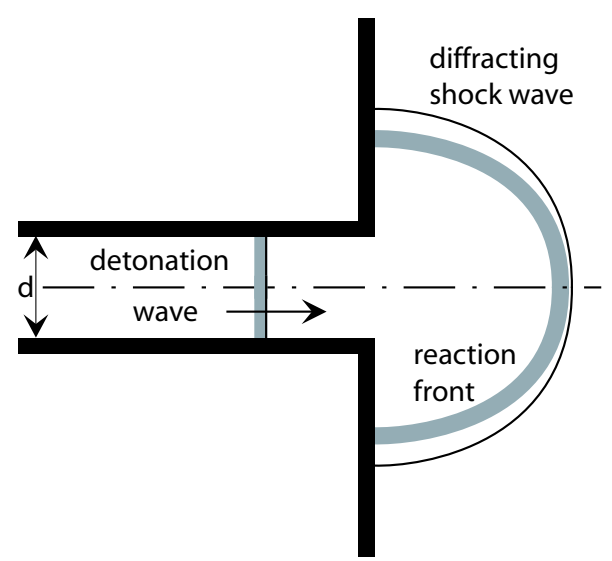

a)

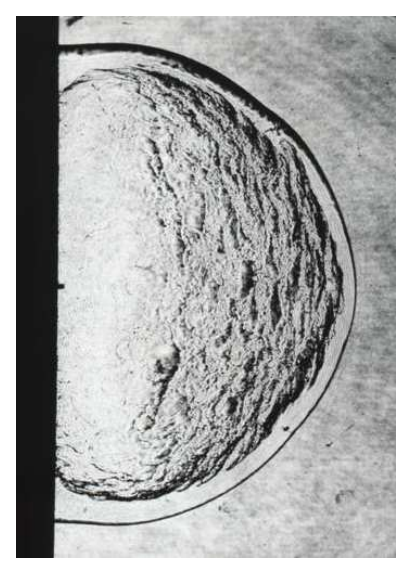

b)

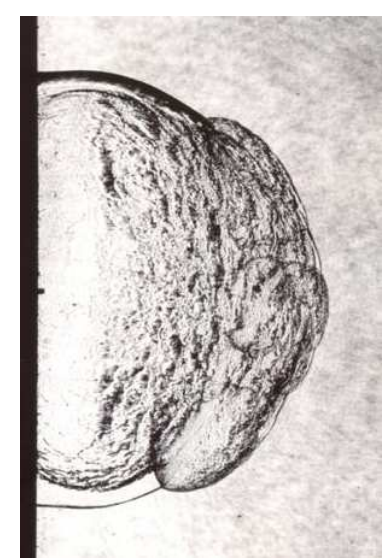

c)

Figure 1: a) Sketch of a detonation wave diffracting out of a circular tube. b) Sub-critical outcome, $\mathrm{H}_{2}+\mathrm{N}_{2} \mathrm{O}, P_{0}=42.5 \mathrm{kPa}$. c) Super-critical outcome, $\mathrm{H}_{2}+\mathrm{N}_{2} \mathrm{O}, P_{0}=45 \mathrm{kPa}$. (b) and (c) Schlieren images, image heights: $125 \mathrm{~mm}$, tube diameter: $38 \mathrm{~mm}$, flow from left to right.

cellular detonation structure.

A variety of optical techniques such as streak imaging, open shutter photography and high-speed schlieren imaging $[1,4]$ has been used to visualize the diffraction process in detonations. To overcome the integrating nature of these visualization techniques and also to allow for sooted foil records, many diffraction experiments in the past were carried out in narrow channels, studying detonation transition from planar to cylindrical geometry. While this enables a better interpretation of the images, the detonation structure remains three-dimensional (3-D) and the transverse waves reflecting from the side walls could influence the diffraction process especially in the critical regime. In this intermediate regime, the re-ignition and failure processes are very sensitive to small perturbations in initial conditions, which are beyond experimental control. The results shown in the present paper are part of a more comprehensive study [8] which investigated qualitatively the spherical diffraction process of detonation waves in various mixtures using schlieren, Planar Laser Induced Fluorescence (PLIF) of the OH radical, multiple exposure chemiluminescence imaging, and stereoscopic image reconstruction of the transverse detonation. The stereoscopic image reconstruction is described in the present paper.

The transverse detonation is a phenomenon of the detonation diffraction process which occurs in the event of re-ignition. The term "re-ignition" itself is misleading since no failure of the entire detonation need precede the re-initiation. It is more a local 


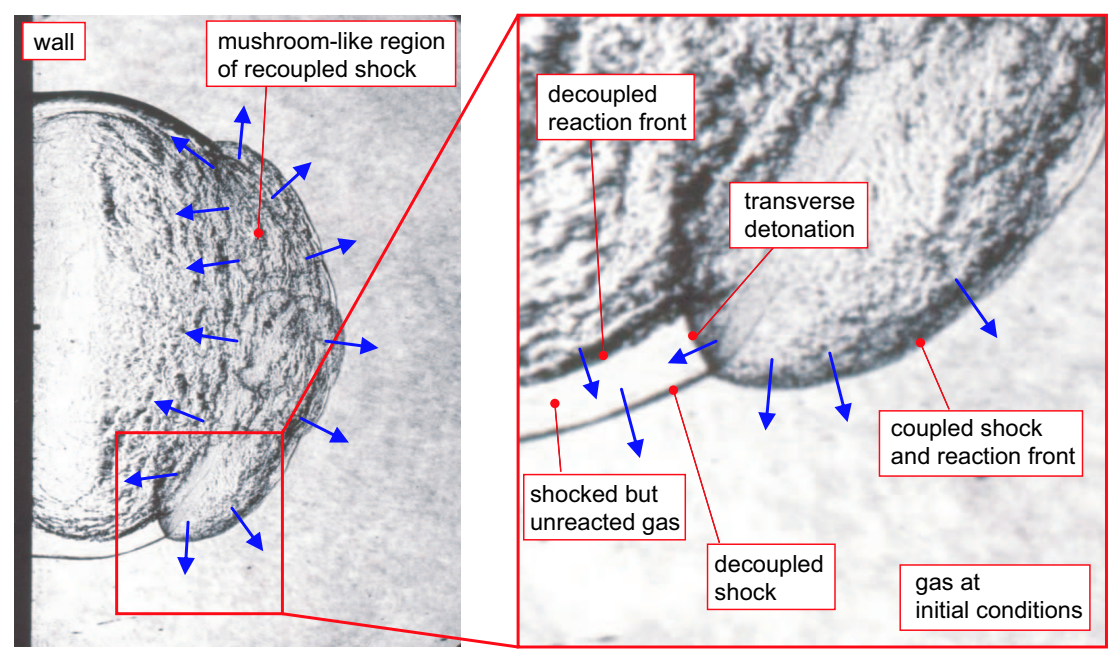

Figure 2: Re-initiation event and detailed view of transverse detonation, Shot $143,0.5 \mathrm{H}_{2}$ $+0.5 \mathrm{~N}_{2} \mathrm{O}, \mathrm{P}_{0}=45 \mathrm{kPa}$. Flow is left to right.

re-coupling of the shock and reaction front. Prior to a re-ignition event, the shocked but unburned reactants are located in a region best described as a thick spherical shell (Fig. 2). The inner radius corresponds to the distance from the tube exit plane center to the reaction front, and the outer radius corresponds to the distance to the lead shock front. The shell thickness varies and is largest close to the wall. Both radii increase with time since the shock and reaction front are progressing outward. In a re-initiation event, a detonation advances transversely through the shocked reactants in the azimuthal and polar directions and completes the reaction in the shell-like region. A growing mushroomlike region of the re-coupled leading shock is created (Fig. 2).

On schlieren images, the transverse detonation resulting from a re-initiation event is best visualized if located on the very top or bottom, since the 3-D masking effect is smallest there. A change in slope and curvature appears in the lead shock at the location of the transverse detonation. Multiple re-ignition events were observed to occur simultaneously at different locations. On chemiluminescence images, the transverse detonations appear as bright bands, as the transverse detonation travels into the pre-shocked gas, resulting in a higher temperature and higher density than in the case of the detonation propagating into the gas of initial mixture conditions (Fig. 7a and b). It is difficult to locate exactly the structure of the transverse detonation and origin of re-initiation from a single image. In order to obtain further insight into the transverse detonation and also its position with respect to the shock surface, the stereoscopic image reconstruction was 


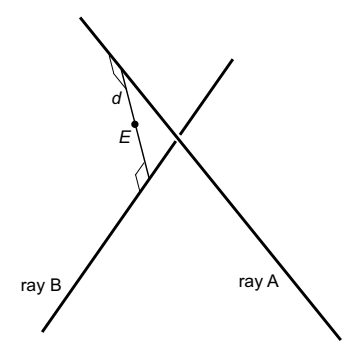

a)

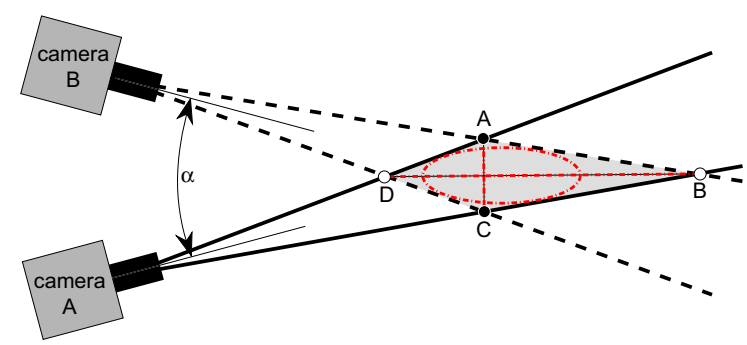

b)

Figure 3: a) Illustration of a skew ray pair from camera $\mathrm{A}$ and $\mathrm{B}$ at a distance $d$ apart and location of center point $E$. b) Principle of stereoscopic imaging of two particles located at $\mathrm{A}$ and $\mathrm{C}$ and their corresponding ghost images located at $\mathrm{B}$ and $\mathrm{D}$.

performed.

\section{General Considerations}

A brief description of stereoscopic imaging is given at the beginning since it is crucial for an understanding of the limitations of the imaging technique in this particular application. In order to reconstruct the 3 -D position of a single particle, the images of two cameras are sufficient, as long as their line of sight is not collinear. A 3-D ray pair corresponding to the particle images can be assigned to each image. The ray pair intersects in the ideal case, and the intersection point determines the 3-D particle location. Due to the digital nature of the image and errors in the camera parameters and calibration, the calculated ray pair does not intersect. Instead, the ray pair is skew (Fig. 3a). In this case, the closest point $E$ to both rays is often taken as the reconstructed 3-D position. This point is located half-way between the line describing the shortest distance $d$ between both rays. Sub-pixel resolution and careful calibration can minimize that distance.

If two ideal point particles are in the field of view and both ray pairs assigned to each particle are within one plane, the ray pairs intersect more than twice (Fig. 3b). This leads to ghost particles in the reconstruction process. The principal rays of both cameras are at an angle $\alpha$. Assuming that the actual positions of the particles are at points $\mathrm{A}$ and $\mathrm{C}$, ghost particles occur during the image reconstruction at positions $\mathrm{B}$ and $\mathrm{D}$. In this case, it is not possible to retrieve the actual 3-D position with two cameras, and the particles could be located either at $\mathrm{A}$ and $\mathrm{C}$ or $\mathrm{B}$ and D. If an ideal line connecting points $\mathrm{A}$ and $\mathrm{C}$ is imaged, the possible line locations, which are reconstructed from the ray tracing, are not just the straight line connecting $\mathrm{B}$ and $\mathrm{D}$. Any line or 2-D object 
within the tetragon $\mathrm{ABCD}$ could result in the same set of images as long as each tetragon side is in contact with the object at least once, as indicated by the ellipse fitted in the shaded region (Fig 3). The region describing the possible locations of the imaged object extends far into the direction of the principle rays for both small $\alpha$ and $\alpha$ close to $180^{\circ}$.

This 3-D reconstruction technique is used to visualize the transverse detonation as defined by the volume in space with high luminosity. We do not attempt to resolve the spatial variations, since for a complex geometry, this is not possible with only two cam-

eras. Instead, the 3-D location is reconstructed of those points with chemiluminescence exceeding a certain level. The reconstruction technique is based on gradients, in contrast to the techniques based on target points as used, for example, in 3-D particle image velocimetry [6]. This is advantageous in the present case since the transverse detonation appears as a relatively thin band, limiting the possible error in the depth of scene. As shown in Fig 3, a large distance between points $\mathrm{A}$ and $\mathrm{C}$ results in a very large distance between $\mathrm{B}$ and $\mathrm{C}$. This issue is addressed further below as an actual example of 3-D reconstruction is discussed, illustrating the limitations and step-by-step reconstruction procedure.

\section{Experimental Setup}

The detonation diffraction facility consists of a $1.5 \mathrm{~m}$ long tube with an inner diameter of $38 \mathrm{~mm}$, which is attached to the $0.8 \mathrm{~m}$ long test section with a $152 \mathrm{~mm}$ square cross section (Fig. 4), described in more detail in [8]. The tube end face plate attached to the diffraction tube inside the test section creates a rotationally symmetric sharp concave corner of $90^{\circ}$. Prior to an experiment, the facility was evacuated, filled with the mixture components by the method of partial pressures, and, finally, circulated for eight minutes to ensure a uniform mixture. The images for the stereoscopic reconstruction were obtained from a diffraction experiment with a stoichiometric $\mathrm{C}_{2} \mathrm{H}_{4}-\mathrm{O}_{2}$ mixture at an initial temperature and pressure of $T_{0}=298 \mathrm{~K}$ and $p_{0}=42.5 \mathrm{kPa}$. The chemiluminescence intensity for this mixture type was highest among the mixtures studied [8].

The detonation is initiated by an electrical spark and a Shchelkin spiral for enhancing the deflagration-to-detonation transition. The detonation wave speed was determined by time of flight data obtained from six pressure transducers placed in the tube and test section. The wave speed in the tube was within $1.5 \%$ of the calculated Chapman-Jouguet 


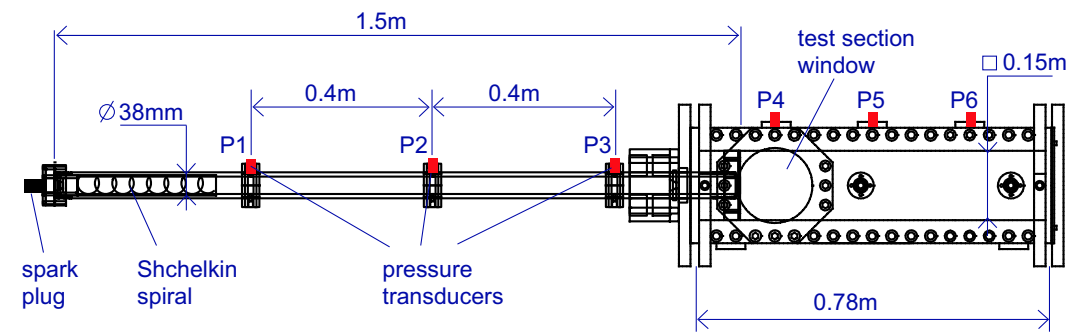

Figure 4: Schematic of detonation diffraction facility.

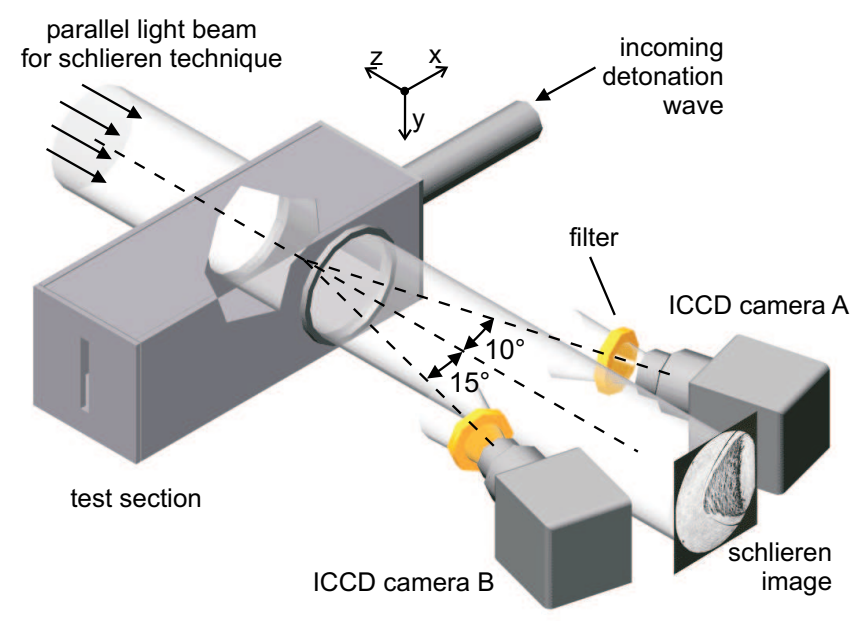

Figure 5: Experimental setup for stereoscopic and schlieren imaging.

(CJ) detonation velocity. The test section is equipped with two windows, each $64 \mathrm{~mm}$ thick and $150 \mathrm{~mm}$ in diameter. These are located opposite each other, $0.5 \mathrm{~m}$ from the end plate. This allows the parallel schlieren light beam to enter and exit the test section (Fig. 5). Two ICCD cameras were positioned horizontally at an angle of $15^{\circ}$ and $10^{\circ}$ to the optical axis of the schlieren system at a distance of $1350 \mathrm{~mm}$ from the tube center axis (Fig. 5). The angle of $25^{\circ}$ was the maximum that could be achieved without blocking the field of view by the frame of the test section window. Camera A was a $576 \times 384$ pixel 12-bit intensified CCD-Camera (Princeton Instruments ITE/ICCD-576). The image is formed by a $105 \mathrm{~mm} \mathrm{f/16}$ lens (Nikon UV-Nikkor). On camera B, a $105 \mathrm{~mm}$ f/32 Micro Nikkor lens (Nikon) was used to acquire the image via an ICCD assembly (Princeton Instruments PI Max) with a resolution of $512 \times 512$ pixel and a 16-bit dynamic range. The height of the field of view was $125 \mathrm{~mm}$ and a WG305-UV high pass filter was placed in front of each camera to ensure a similar spectral response of both camera systems. The wavelength range detected by both cameras was from $305 \mathrm{~nm}$ to approximately $800 \mathrm{~nm}$. Both cameras were gated simultaneously (within $5 \mathrm{~ns}$ ) and the gate width was $200 \mathrm{~ns}$. 


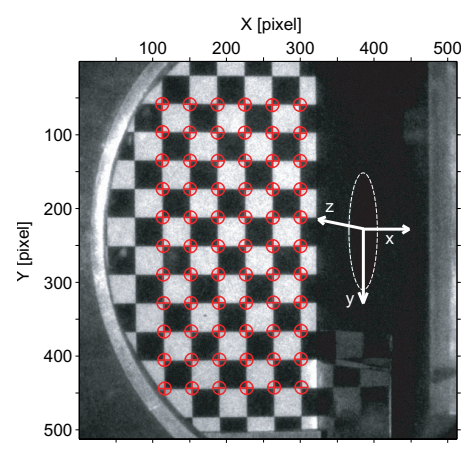

a)

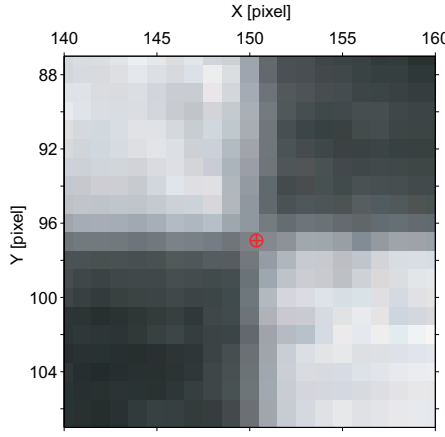

b)

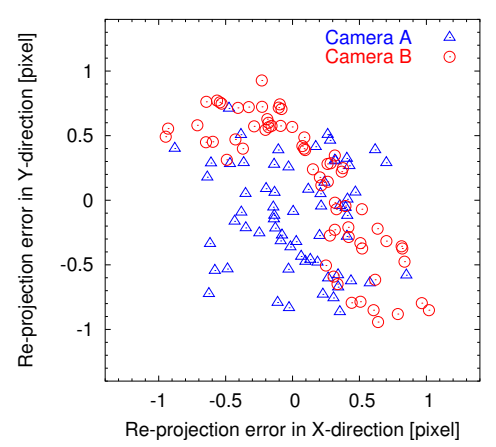

c)

Figure 6: a) Corner-detected image of target placed in $z=60 \mathrm{~mm}$ plane. Units of the photographic coordinate system $(X, Y)$ are pixels. The absolute coordinate system axes are denoted $x, y$, and $z$. The tube exit is indicated by the dashed ellipse b) Section of corner-detected image enlarged to show sub-pixel accuracy of corner finding method. c) Re-projection error for camera target.

\section{Camera Calibration}

The purpose of the camera calibration is to determine the 3-D ray which corresponds to a specific pixel coordinate on each camera. Two images of a calibration target were taken for each camera. The plane checkerboard target with a pattern length of $10 \mathrm{~mm}$ was placed vertically close to the test section side wall facing the cameras $(z=-60 \mathrm{~mm})$. The coordinate system origin is at the center point of the exit plane, and the $x$ axis coincides with the tube axis (Fig. 5 and 6a). The second target position corresponds to $z=60 \mathrm{~mm}$, at the rear of the volume of interest. The general idea about the $3-\mathrm{D}$ ray construction method applied here is that the $3-\mathrm{D}$ ray corresponding to a specific pixel of a camera image is found by reconstructing the absolute coordinates of the points corresponding to that pixel in both target planes. Only the $x$ and $y$ coordinates within the target plane have to be determined since the $z$ coordinate is already given by the target plane itself. The ray is then described by the absolute coordinates of two points, one of them in each target plane. This calibration process is done for both cameras independently.

In order to obtain the absolute (lab) coordinates of the point $P(x, y)$ corresponding to any photographic coordinate $(X, Y)$ on the images, a transformation function has to be determined. Here, the formulas given in [6] are used:

$$
x=\left(c_{1}+c_{2} X+c_{3} Y\right) /\left(1+c_{7} X+c_{8} Y\right),
$$




$$
y=\left(c_{4}+c_{5} X+c_{6} Y\right) /\left(1+c_{7} X+c_{8} Y\right)
$$

The eight camera parameters $c_{i}$ are valid for only one camera and one target position. The total calibration of the stereoscopic system consists of four sets of $c_{i}$ parameters. Each set of calibration parameters was determined by a least squares fit of 66 pattern corner points for which the absolute coordinates $(x, y)$ and photographic coordinates $(X, Y)$ were known. The absolute coordinates of the corner points are given directly by the pattern of the checkerboard whereas the corresponding $X$ and $Y$ were found by digital image processing of the target image. A routine based on the Harris corner finding method [2] was used to extract the corner location for the set of 66 points with sub-pixel accuracy (Fig. 6a and b). Each set of $c_{i}$ parameters together with Eqs. 1 defines the transformation of photographic coordinates into absolute coordinates for a specific target position and camera location. The parameter set was saved in two $2 \times 8$ matrices $\mathbf{C}_{\mathbf{A}}$ and $\mathbf{C}_{\mathbf{B}}$, corresponding to camera $\mathrm{A}$ and $\mathrm{B}$. The accuracy of the functional fit of Eqs. 1 can be tested by re-projection of the corner coordinates. For this calculation, Eqs. 1 is inverted and the set of 66 absolute $(x, y)$-coordinates of the pattern corners is mathematically projected onto the image plane. The inverse transformation is given by

$$
\begin{aligned}
X & =\frac{\left(c_{4} c_{8}-c_{6}\right) x+\left(c_{3}-c_{8} c_{1}\right) y+\left(c_{6} c_{1}-c_{4} c_{3}\right)}{\left(c_{7} c_{6}-c_{8} c_{5}\right) x+\left(c_{2} c_{8}-c_{3} c_{7}\right) y-\left(c_{2} c_{6}-c_{3} c_{5}\right)}, \\
Y & =\frac{\left(c_{5}-c_{7} c_{4}\right) x+\left(c_{1} c_{7}-c_{2}\right) y-\left(c_{1} c_{5}-c_{2} c_{4}\right)}{\left(c_{7} c_{6}-c_{8} c_{5}\right) x+\left(c_{2} c_{8}-c_{3} c_{7}\right) y-\left(c_{2} c_{6}-c_{3} c_{5}\right)} .
\end{aligned}
$$

The corner location in the image plane is then compared to the locations which were previously obtained from the image with the corner finding method. The distance in pixels between the calculated and corner extracted location is the re-projection error (Fig. 6c) and has an $X$ and $Y$ component. The maximum re-projection error is one pixel. The error could be attributed to lens aberrations, refraction through the thick glass window, imperfections in the target, and errors in the digital corner finding method.

\section{$6 \quad$ Image Processing}

The transverse detonation appears as a bright band on the chemiluminescence images (Fig. 7) and must be extracted from other features visible in the image to obtain a set of pixels used for the ray tracing procedure. One possibility is to threshold the image, leading to a binary (black and white) image. The white pixels would correspond to the 


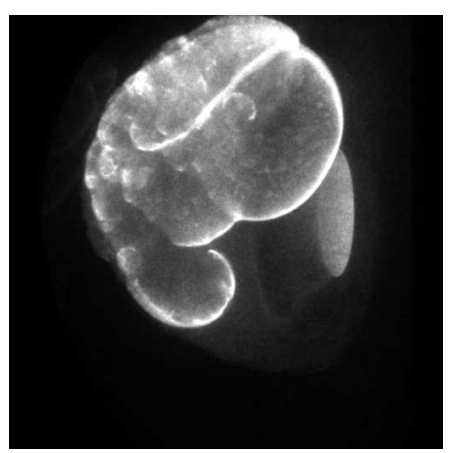

a) Camera B

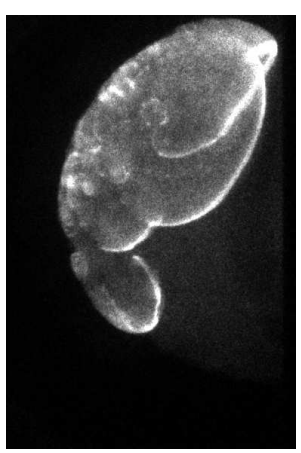

b) Camera A

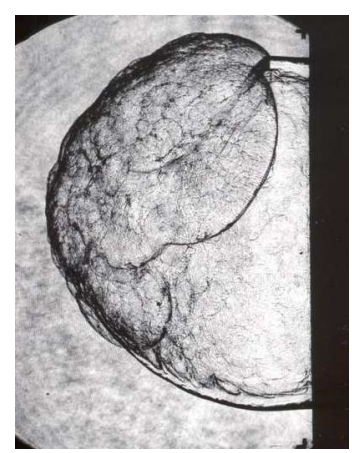

c) schlieren

Figure 7: a) and b) Chemiluminescence images of camera A and B for shot 223, $\mathrm{C}_{2} \mathrm{H}_{6}+$ $3.5 \mathrm{O}_{2}, \mathrm{P}_{0}=42.5 \mathrm{kPa}$. Flow direction is right to left. c) Simultaneously obtained schlieren image. Tube end plate is located to the right.

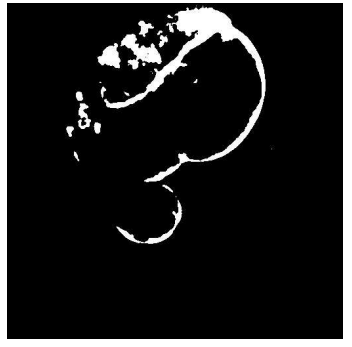

a) threshold: 0.72 .

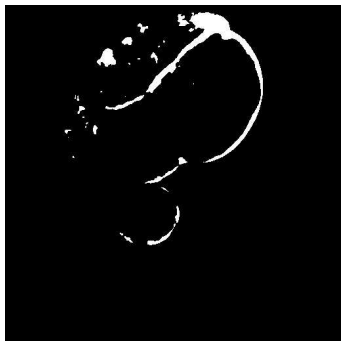

b) threshold: 0.84 .

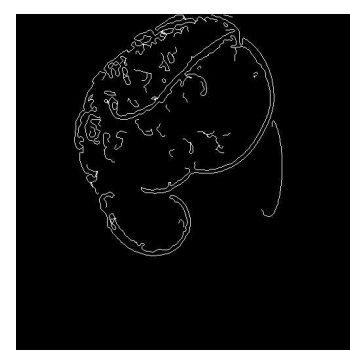

c) edge detected

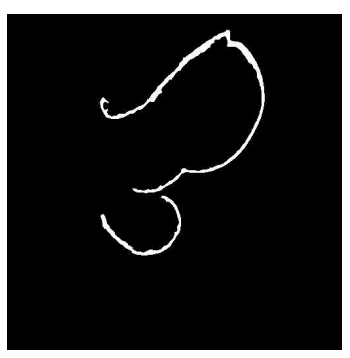

d) final

Figure 8: a) and b) Thresholded chemiluminescence images for two different threshold values. The images were prior to thresholding blurred by a Gaussian filter with a radius of 2 pixel. c) Laplacian edge detected image, which was blurred with a median filter prior to processing. d) Processed and filled edge detected image used for 3-D image reconstruction.

transverse detonation. This technique did not isolate the bright band sufficiently from the background over the entire image, since the chemiluminescence intensity of the band did not remain constant. For lower threshold values (Fig.8a), the details of the brighter region on the image top were not resolved; for higher threshold values (Fig. 8b), the transverse detonation on the bottom was not fully detected and another image processing method was needed.

Since the transverse wave appears as a local intensity gradient on the images, we used an edge detection routine in conjunction with the thresholded images. The image was filtered by a $5 \times 5$ pixel median filter prior to the edge detection; this suppresses impulse noise while preserving strong gradients. The blurred image was then edge detected with the zero-crossing Laplacian edge detector. The edge detector threshold was set manually. 


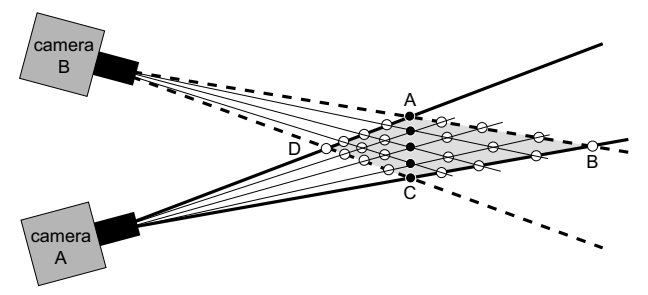

Figure 9: Closely spaced ray bundles lead to a point cloud in the reconstruction process.

The edge-detected image included, besides the edges along the transverse detonation, the edges of the overall leading luminosity front and other undesired structures seen in Fig. 8c. To minimize the manual deletion of these edges, the edge-detected image was multiplied pixel by pixel by the thresholded image. In a further step, end-points of edge segments with a distance smaller than eight pixels were connected by straight lines in order to obtain a closed edge line enclosing the bright region. For some small regions, the outline had to be manually traced before the last step, the binary flood-fill operation, was performed. The final result (Fig. 8d) was an image that contained only information from the transverse detonation. In the 3-D reconstruction process, the transverse detonation location corresponds to the set of white pixels on the processed image.

\section{Reconstruction Process}

The set of photographic coordinates $X$ and $Y$ corresponding to the white pixels of the processed images of camera $A$ and $B$ were stored in two separate matrices, $\mathbf{P}_{\mathbf{A}}(5127 \times 2)$ and $\mathbf{P}_{\mathbf{B}}(6345 \times 2)$. The absolute coordinates of the points in the front and back target plane corresponding to $\mathbf{P}_{\mathbf{A}}$ can be calculated via Eqs. 1 and the set of camera parameters $\mathbf{C}_{\mathbf{A}}$. For the image taken by camera A, this results in a set of $5127 \times 6$ coordinates representing the $(x, y, z)$-coordinates in the front and back target planes. The $z$-coordinate is either $-60 \mathrm{~mm}$ or $60 \mathrm{~mm}$, the location of the target planes. This set of point pair coordinates defines the 3-D ray to each white pixel and is stored for image $\mathrm{A}$ in $\mathbf{R}_{\mathbf{A}}$ $(5127 \times 6)$ and, correspondingly for image $\mathrm{B}$, in $\mathbf{R}_{\mathbf{B}}(6345 \times 6)$. The rays are saved in the two-point form as two points define a straight ray, which is represented parametrically through the test section. Up to this point, the data from both camera images were processed independently.

In order to decide which ray of $\mathbf{R}_{\mathbf{A}}$ correlates with which ray of $\mathbf{R}_{\mathbf{B}}$, the distance $d$ (Fig. 3a) between every possible ray pair, one ray each from $\mathbf{R}_{\mathbf{A}}$ and $\mathbf{R}_{\mathbf{B}}$, was calculated 

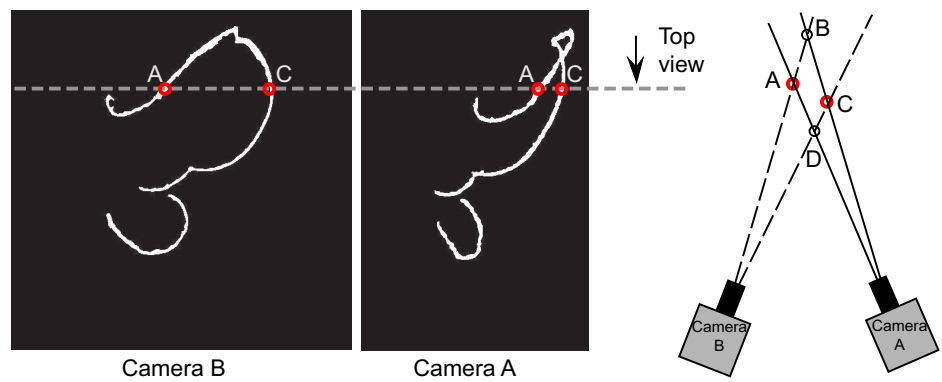

Figure 10: Effect of multiple intersections of the transverse detonation geometry with the camera plane.

and stored in matrix D $(5127 \times 6345)$. Ideally, rays which correspond exactly to a single point in space would intersect, but all rays were found to be skew for the calculation precision of 16 significant digits used. This "skewness" is due to various sources of errors mentioned earlier and is inevitable in 3-D image reconstruction from real 2-D images. The distance $d$ between rays is a measure of both the correction to a given point in space and the skew errors. The maximum $d$ calculated was $74 \mathrm{~mm}$, which is a large error and the two rays are very unlikely to arise from the same region of chemiluminescence. This ray pair is actually one ray on the very top of the image $A$ and one from the very bottom of image B. The smallest distance $d$ found was calculated to be $3 \mathrm{~nm}$, which indicates that it is almost certain that these two rays arise from the same location in the test section. Note that even if $d=0$, the rays do not have to arise from the same location in the test section, since, for example, the ray from camera A could arise from point A and the ray from camera $\mathrm{B}$ could arise from point $\mathrm{C}$ and both intersect ideally in point D (Fig. 9). The decision regarding which ray pairs are considered as correlated can be based on the distance matrix $\mathbf{D}$. All ray pairs for which $d$ is below a certain threshold are assumed to correlate. Note that one ray from image A can be correlated with multiple rays of image $B$ since the chemiluminescence intensity of the imaged volume is integrated over the line of sight of each camera and the intensity information is lost by the edgedetection processes of the image. This is similar to the situation in particle tracking for which several particles are aligned and appear on one camera as a single point but multiple points on the other camera. For the example given in Fig. 3b, this means that the particle positions are reconstructed at all four locations, A, B, C, and D.

This effect influences the reconstruction process on two levels, depending on the distance between the ambiguous points. The case of a large distance between ambiguous points $\mathrm{A}$ and $\mathrm{C}$ is shown on Fig. 10. The transverse detonation image crosses the hori- 


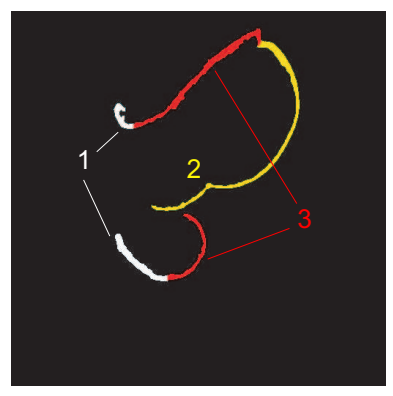

a) Camera B

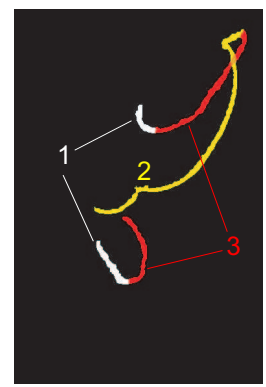

b) Camera A

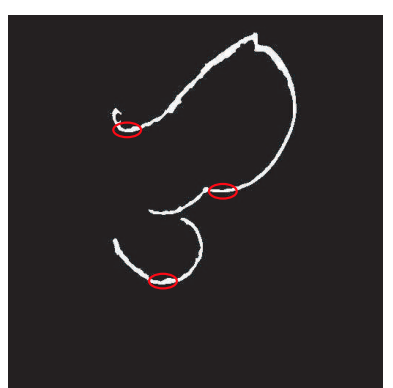

c)

Figure 11: a) and b): Manual sectioning of corresponding branches on both images. c) Regions in which the horizontal extent of detected pixel is large.

zontal plane described by the cameras' principle rays more than once. From the context, it is obvious which section of the transverse detonation on image A corresponds to which one on image B. For the dashed plane shown in Fig. 10, it is clear that the left branch seen by camera $\mathrm{B}$ corresponds to the left branch seen by camera $\mathrm{A}$. In the reconstruction (Fig. 10 right), the correct location is $\mathrm{A}$ and $\mathrm{C}$ - not $\mathrm{B}$ and $\mathrm{D}$. If the locations were $\mathrm{D}$ and $\mathrm{B}$, corresponding branches would occur on opposite sides on the images. To overcome this problem in the automated image reconstruction, the entire transverse detonation band was divided up on both images into three slightly overlapping segments (Fig. 11), which, by visual inspection, correspond to each other. The distance $d$ between rays of different segments was set to a large number to avoid triggering spurious reconstruction points.

If the ambiguous points are close together (Fig. 9), a bundle of dense rays from one camera is detected to correlate with a bundle of rays from the other camera. The reconstructed locations will, in this case, correspond to a point cloud. The underlying effect is the same as discussed in the previous paragraph in the context of segmenting of the image, just on a smaller scale. The volume of the point cloud gets created in the reconstruction process. This effect is observed as the band of higher intensity that is several pixels wide and results in elongation of the reconstructed volume in the principal ray direction.

During the calculation of the distance matrix $\mathbf{D}$, the absolute coordinates $(x, y, z)$ of the center point $E$ of the shortest line segment (Fig. 9a) between all rays from image A and all rays from image $B$ were stored in a matrix $\mathbf{E}(5127 \times 6345 \times 3)$. The matrix $\mathbf{E}$ can be thought of as a look-up table for the locations of a chemiluminescence "event", 


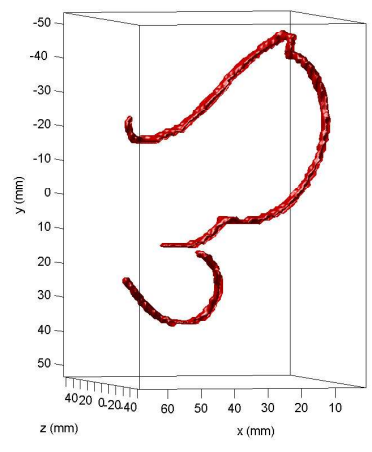

a) Camera $B$

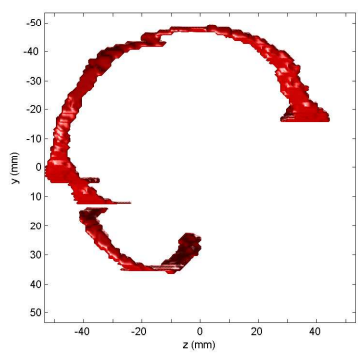

d) Back

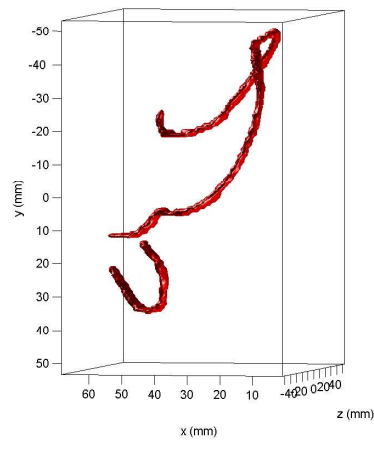

b) Camera A

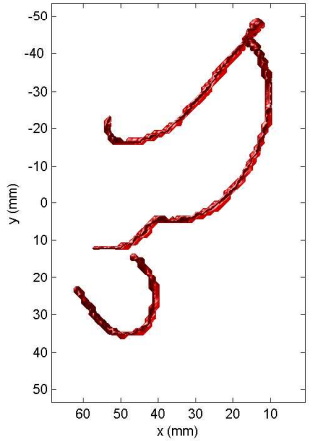

c) Side

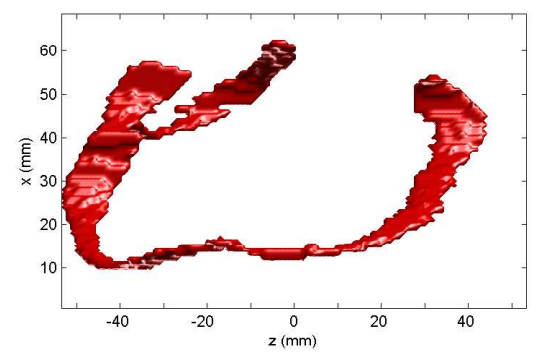

e) Top

Figure 12: Reconstructed 3-D iso-surface corresponding to different view points.

given a specific ray pair. Together with $\mathbf{D}$, this enables the plotting of point clouds which correspond to a certain maximum distance $d_{\text {err }}$. However, point clouds are difficult to interpret in 3-D plots. Iso-surfaces that correspond to a certain distance $d_{e r r}$ are easier to view and interpret. In order to create iso-surfaces, the data have to be projected on an orthogonal grid.

The iso-surface is a closed surface for which all points inside this surface correspond to $d<d_{e r r}$. An orthogonal equidistant grid of vertex length $0.5 \mathrm{~mm}$ was introduced to cover the volume of interest $(70 \mathrm{~mm} \times 120 \mathrm{~mm} \times 120 \mathrm{~mm})$ in the test section. To each vertex point $(x, y, z)$, the scalar value of the minimum $d$ found within the cubic volume around the vertex was attached. The iso-surface for $d_{e r r}=0.1 \mathrm{~mm}$ was found to give good results as the thickness of the reconstructed region (Fig. 12) corresponds visually to the thickness of the bright region of the transverse detonation seen on the original images (Fig. 7). Iso-surfaces for a much smaller value of $d$ appeared punctured with openings while, for larger values of $d$, the extent in the field of view direction becomes very large. The 3-D image was manually post-processed in regions in which the horizontal extent of detected pixel was large (Fig. 11c). In these regions, the iso-surface extended far into 


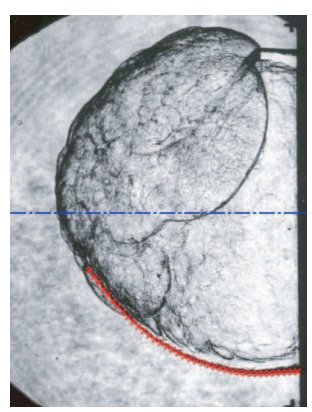

a)

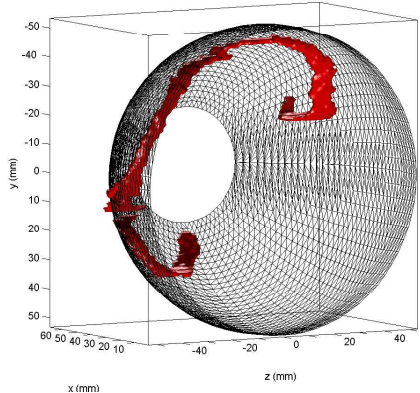

b)

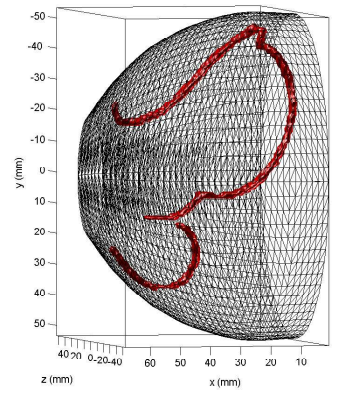

c)

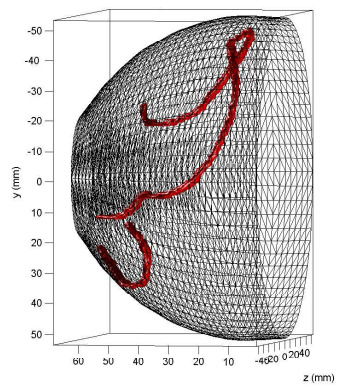

d)

Figure 13: a) Edge-detected part of leading shock (red) and assumed axis of symmetry. Flow direction right to left. b)-d) Reconstructed decoupled shock surface and transverse detonation. b) View from rear of the wave through the tube exit plane. c) View corresponding to camera B. d) View corresponding to camera A, tube exit plane located on the right.

the depth of scene direction and was cropped manually. A larger angle between the two cameras or a third camera would minimize this limitation. In the regions where the band of higher luminosity on the chemiluminescence images is close to being horizontal, one can observe the larger extent in the $z$-direction, which corresponds to the viewing direction of the cameras (Fig. 12d).

\section{Reconstruction Of Shock Surface}

In order to allow for a determination of the transverse detonation location with respect to the leading shock, the simultaneously obtained schlieren image was analyzed. The region of the completely decoupled shock, which can be assumed to be axisymmetric, was edge detected (Fig. 13a). The derived shock surface is therefore not closed but open in the very front where axial symmetry could not be assumed. The shock surface was then plotted on the same coordinate system as the transverse detonation. This enabled locating the position of the transverse detonation location with respect to the shock surface, shown as a grid in Fig. 13b, c and d.

Discounting some artifacts arising from the relatively small angle between the cameras, the transverse detonation can be clearly located just below the decoupled shock. In some locations, the reconstructed iso-surface of the transverse detonation is found outside the reconstructed shock surface, which can probably be explained by the large uncertainty of the iso-surface in the $z$-direction. Other possible error sources include 
those mentioned earlier with regard to the calibration process and image processing. The reconstructed transverse detonation is divided into two separate parts. The upper part of the transverse detonations has two kinks at $(x, y, z)=(40,5,-40)$ and $(15,-45,-15)$ (see Fig. 12c). These kinks indicate junctions between transverse detonations, which originated from different re-ignition points. Three independent points of re-ignition seem to have caused the geometry of the upper part of the transverse detonation. The lower part of the transverse detonation appears smooth and seems to originate from a single re-ignition event.

\section{Conclusions}

The image 3-D reconstruction process clearly reveals the $3-\mathrm{D}$ nature of the transverse detonation. The transverse detonation progresses into the shocked but unreacted fluid, which is located under the decoupled shock wave. The structure of the transverse detonation indicates the presence of several independent re-ignition locations. To allow for smaller errors in the reconstructed transverse detonation geometry, especially in the $z$ direction, which is the main limitation in the current setup, a larger angle between the two cameras or a third camera is needed. If only one camera is available a mirror could be placed on the opposite wall of the windows to allow for a second view angle on the same image. As the path length to the imaged object varies, a small $f$-number will be needed with a mirror for both images to be in focus. The back window in the current setup reflects some of the chemiluminescence intensity, but the reflected light intensity is too low to allow for a $3-\mathrm{D}$ reconstruction based on these reflections.

\section{References}

[1] D.H. Edwards, G.O. Thomas, and M.A. Nettleton, The diffraction of a planar detonation wave at an abrupt area change, Journal of Fluid Mechanics 95 (1979), 79-96.

[2] C.G. Harris and M. Stephens, A combined corner and edge detector., In Proc. of the 4th Alvey Vision Conference (Manchester, UK), 1988, pp. 147-151.

[3] D.A. Jones, G. Kemister, E.S. Oran, and M. Sichel, The influence of cellular structure on detonation transmission, Shock Waves 6 (1996), 119-129. 
[4] V.V. Mitrofanov and R.I. Soloukhin, The diffraction of multifront detonation waves, Soviet Physics-Doklady 9(12) (1965), 1055-1058.

[5] S.B. Murray and J.H. Lee, On the transformation of planar detonation to cylindrical detonation, Combustion and Flame 52 (1983), 269-189.

[6] K. Nishino, N. Kasagi, and M. Hirata, Three-dimensional particle tracking velocimetry based on automated digital image processing, Journal of Fluids Engineering, Transactions of the ASME 111 (1989), 384-391.

[7] E.G. Pantow, M. Fischer, and T. Kratzel, Decoupling and recoupling of detonation waves associated with sudden diffraction, Shock Waves 6 (1996), 119-129.

[8] Florian Pintgen, Detonation diffraction in mixtures with various degrees of instability, Ph.D. thesis, California Institute of Technology, Pasadena, California, Dec 2004.

[9] E. Schultz, Detonation diffraction through an abrupt area expansion, Ph.D. thesis, California Institute of Technology, 2000.

[10] J.E. Shepherd, I.O. Moen, S.B. Murray, and P.A. Thibault, Analysis of the cellular structure of detonations, 21st (Int.) Symposium on Combust., The Combustion Institute, 1986, pp. 1649-1658. 